\title{
The Virtual Prototype Design of Boating Robot Based on ADAMS
}

\author{
Yao Fu \\ College of Engineering, \\ HuaZhong Agriculture University, \\ Wuhan, China \\ azureology@qq.com
}

\author{
Xurong Li \\ College of Engineering, \\ HuaZhong Agriculture University, \\ Wuhan, China \\ 438409371@qq.com
}

\begin{abstract}
This paper completed the virtual prototype design for a boating robot in ADAMS. After adding the joints and motion functions, a dynamic simulation and analysis to the virtual prototype had been carried out. It approved the boating robot could achieve the intended motion as the requirement. The simulation provided a theoretical basis for the further design, which is a reference for the next improving design.
\end{abstract} robot

Keywords-virtual prototyple, dynamic simulation, boating

\section{Introduction}

Along with the reform and opening up, China's economic and the development of industry is rising rapidly. In robot field, there are rickshaw robot, helpmate robot, entertaining robot, cleaning robot and so on. With the development of water tourism project, it has very promising prospects to design a boating robot. The boating robot can not only provide boating entertainment services for some water park, happy valley, scenic spots, but also be used for water transport of goods. On the defined line waters, boating robot has more advantages, such as, labor saving, low error rate, the night working without affecting the efficiency and high security.

The overall structure of the boating robot is shown in Fig.1

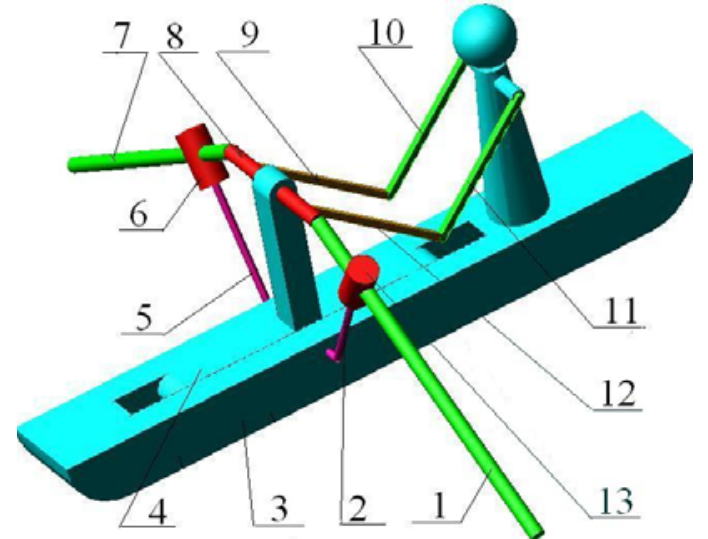

1-oar1 2-support1 3-shipBody 4-moveLink 5-support2 6-link1 7-oar2 8-handle 9-arm1 10-arm2 11-arm4 12-arm3 13-link2

Fig.1 The structure of boating robot.

\section{Set UP THE VIRTUAL PROTOTYPE MODEL OF THE BOATING ROBOT}

Click Settings $\rightarrow$ unit $\rightarrow$ MMKS. Working Gird $\rightarrow$ set Size $\mathrm{X}=750 \mathrm{Y}=750$, Spacing $\mathrm{X}=20 \mathrm{Y}=20$.Click Settings $\rightarrow$ Icon, set New Size $=30$. Click F4 to open the coordinate window..

\section{A. Create the shipBody}

Click the box icon and set length=920 Width=120, depth $=80$ to create a box. Adjust the box center to the origin of the coordinate. Rename it as shipbody. Click the icon and set radius $=80$. Choose 2 edges. Click the box icon and choose point $(-400,0,0)$ and $(150,0,0)$ to create a box. Click the icon to cut it from the shipBody. Click the icon and choose point $(200,0,0)$ and $(200,200,0)$ to create a frustum. Click the icon $\bigcirc$ and set the radius=50.Click $(200,250,0)$ to create a sphere. Click settings, working grid, set orientation: global YZ. Click the cylinder icon and set the radius=10and click the point $(60,200,0)$ and $(-60,200,0)$ to create a cylinder. Click the icon to union the frustum, sphere. cylinder and shipBody.

\section{B. Create the handle}

Click settings, working grid, set orientation: global YZ Click the cylinder icon and set the radius=10and click the point $(100,220,0)$ and $(-100,220,0)$ to create a cylinder. Rename it as handle.

\section{Create the oar1 and oar2}

Click settings, working grid, set location-pick. Choose the point(-120,-20,0).Click set orientation: global YZ. Click the icon 1 . Click the cylinder icon and set the radius $=10$. Choose the point $(-100,220)$ and $(-540,-20,0)$ to create oar1.Choose the point $(100,220)$ and $(540,-20,0)$ to create oar2.

\section{Create the support1 and support2}

Click the cylinder icon and set the radius $=5$. Choose the point $(-60,0,0)$ and $(-80,0,0)$. Choose the point $(-800,0,0)$ and $(-$ $80,-180,0)$.Click the icon to unite 2 cylinders and rename is as support1.Support2 mirrors to the support1.The same way to create the support2. 


\section{E. Create the link1 and link2}

Click the cylinder icon and set the radius $=20$. Choose the point $(-160,-140,0)$ and $(-200,200,0)$ to create a cylinder and rename is as link1.Create the link2 as the same way.

\section{F. Create the arm1 to arm4}

According to the Fig.1 adjust the view orientation and click the link icon to create the arm1,arm2,arm3 and arm4.

Now the boating robot model is shown as Fig.1.

\section{ADD JOINTS AND MOTIONS}

\section{A. Add revolute joint}

In the Fig.1,there are revolute joints between 2 and 3,5 and 3,5 and 6,2 and 13,8 and 4,8 and 9,8 and 12,9 and 10,11 and 12,10 and 3,11 and 3 .

\section{B. Add the translational joint}

In the Fig.1, there are translational joints between 3 and 4,1 and 13,6 and 7.

\section{Add the spherical joint}

In the Fig.1,there is spherical joint between 1 and 8 .

\section{Add the hook joint}

In the Fig. 1 there is hook joint between 7 and 8.

\section{E. Add motions}

Click general motions icon $\%$ and choose the revolute joint between 5 and 6 . Click general motions icon 2 and choose the hook joint between 8 and 7 . Click the translational joint motion icon and choose the translational joint between 3 and 4.Modify it function as $80 * \sin (1 *$ time $)$.

Now the virtual prototype model of the boating robot is shown in Fig.2.

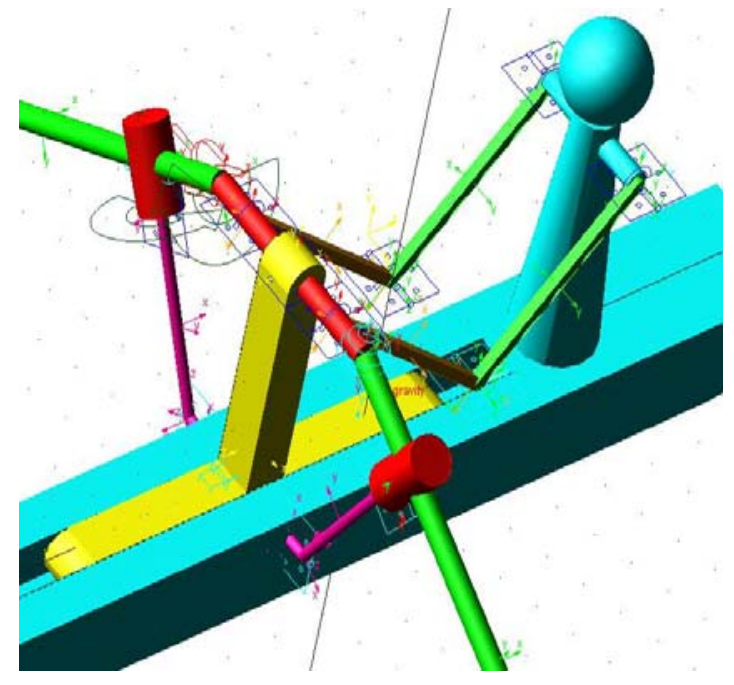

Fig.2 the virtual prototype model of the boating robot

\section{SIMULATION AND ANALYSIS}

In order to show the dynamic simulation, add a box under the boat. It shows in Fig.3.Click the simulation icon 항 and set end time $=25$,steps $=500$. Click the icon $D$ to test the simulation
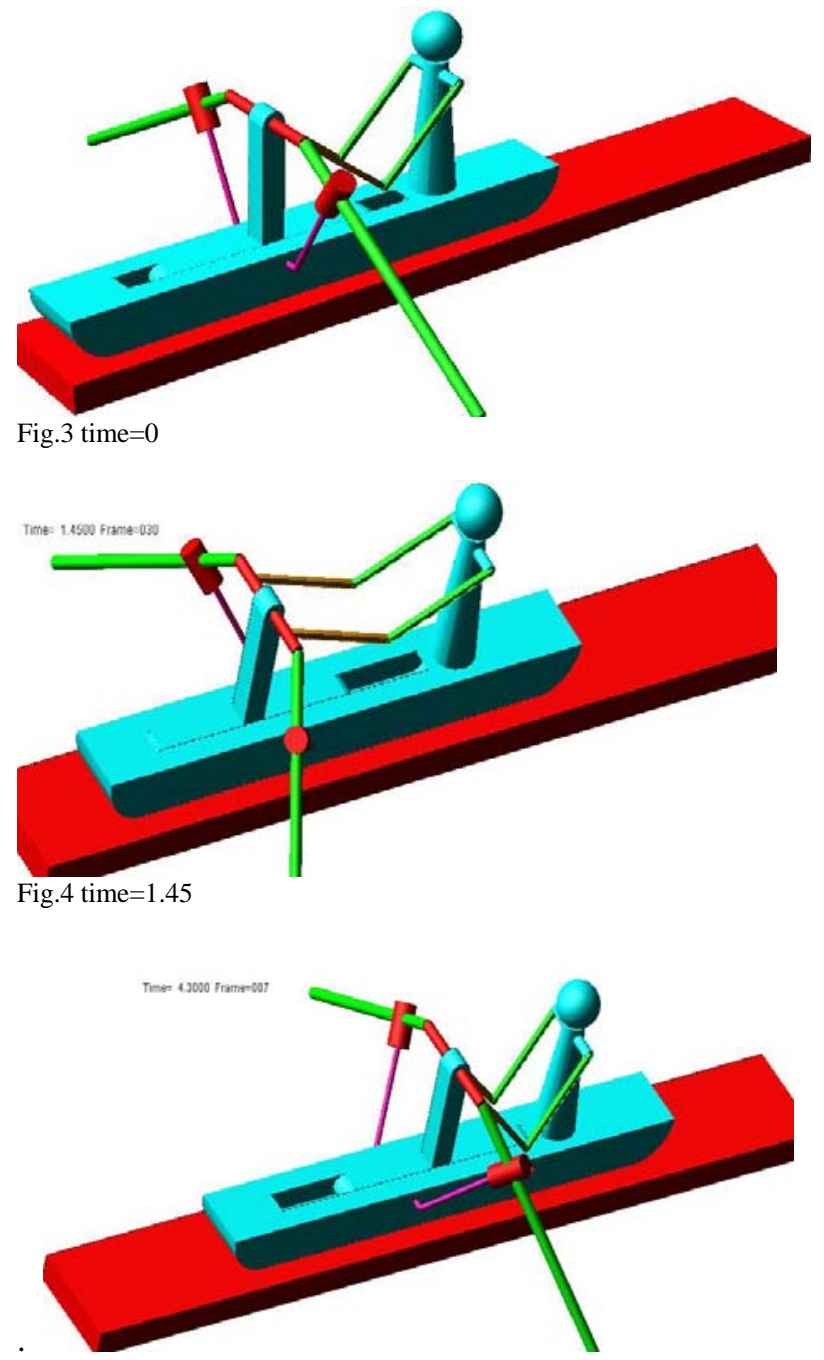

Fig. 5 time $=4.3$

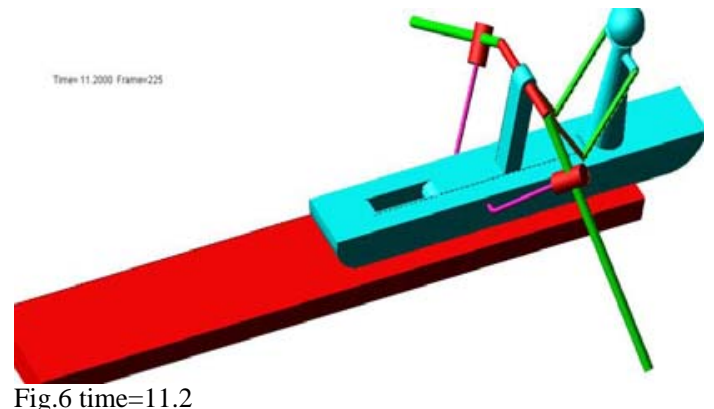

From Fig.3 to Fig.6,it shows the boats different state with different time and the boat is moving by the motion. From Fig.7 to Fig.9,it shows the boat is moving along $x$ direction only. 
From Fig.10 it approves the boat velocity is periodically.Fig.11 shows jiangs angular velocity is periodically too. Fig.12 shows there have been slight oscillations in jiang angular acceleration.

Therefore the boat is moving according to the design requirement.

\section{CONCLUSION}

This paper designs a boating robot according to the requirement to the working on the waters. Meanwhile the virtual prototype modeling of the boating robot is set up. The dynamic simulation and analysis to the boating robot have been carried out. It approves that the boating robot can achieve the intended motion. The design of virtual prototype modeling of the boating robot has been verified as correct.

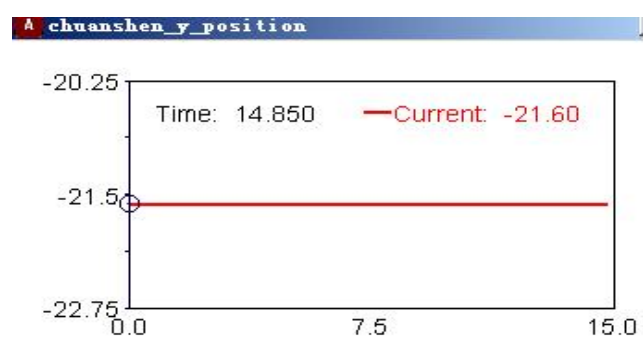

Fig.7 chuanshen y position

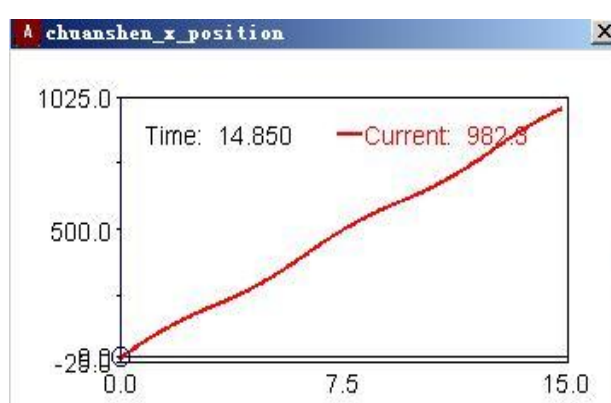

Fig.9 chuanshen $\mathrm{X}$ positon

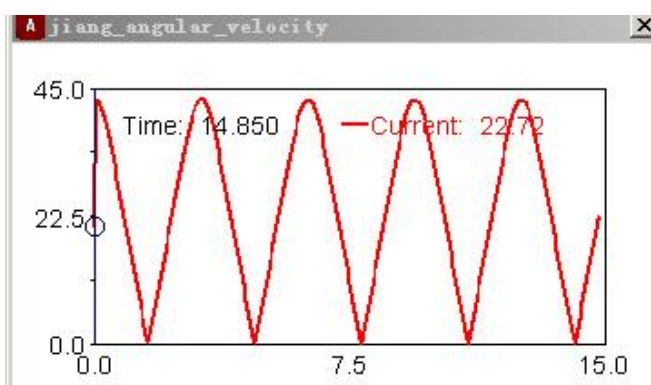

Fig.11 jiang angular velocity

\section{References}

[1] Zenggang Li,An introduction and exaple for ADAMS(National Defense Industry Press, Beijing,china 2007)

[2] Hanyuan Liao, Jianyi Kong, Theory of Machines and Mechanism(Machinery Industry Press,Beijing,china 2007)

[3] Xurong Li, Xiangzhou Zheng,ADAMS-emabled virtual prototyping and dynamic simulation approach into planing mechanism: submiteed to Chinese Journal of Construcion machinery,Vol.5 No.4,2007 Totally 504 P437-439.

[4] Xurong Li,Shouyi Zhang,The Virtual Reality Simulation for the Unmanned Lunar Vechicle Based on ADAMS:submitted to The International Conference on Multimedia Technology totally 1242 P972974 (2010).

[5] Xurong Li,Kundian Wang, Virtual Prototype Design of Spherical Material Vibration Grader Machine Based on ADAMS:submitted to Advanced Research on Intellignent Systems and Mechanical Engineering 644 P271-274(2013).

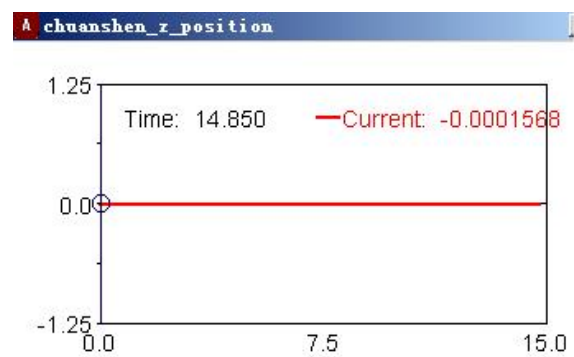

Fig.8 chuanshen z position

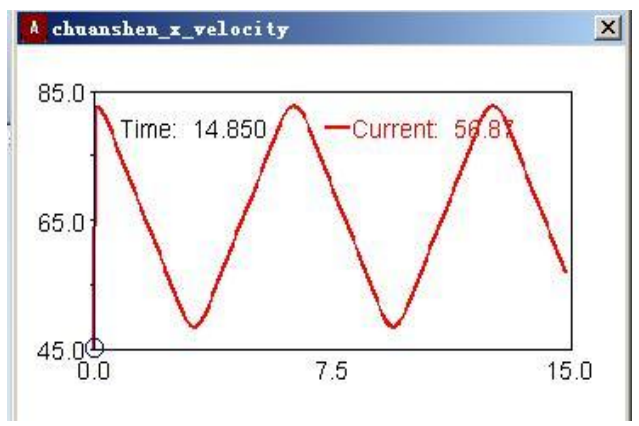

Fig.10 chuanshen x velocity

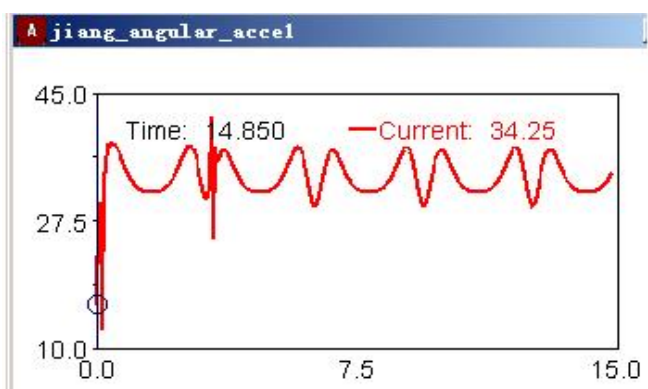

Fig.12 jiang angular acceleration 